Fractures. Craniomaxillofacial Trauma Reconstr Open. 2020;5:2472751220940130. doi:10.1177/2472751220940130

4. Onișor-Gligor F, Tent PA, Bran S, Juncar $M$. A Naso-Orbito-Ethmoid (NOE) Fracture Associated with Bilateral Anterior and Posterior Frontal Sinus Wall Fractures Caused by a Horse Kick-Case Report and Short Literature Review. Medicina (Mex). 2019; 55(11):731. doi:10.3390/ medicina5 5110731

5. Wei J-J, Tang Z-L, Liu L, Liao X-J, Yu Y-B, Jing $W$. The management of naso-orbital-ethmoid (NOE) fractures. Chin J Traumatol. 2015; 18(5):296-301. doi:10.1016/j.cjtee.2015.07.006

6. Potter JK, Muzaffar AR, Ellis E, Rohrich RJ, Hackney FL. Aesthetic management of the nasal component of naso-orbital ethmoid fractures. Plast Reconstr Surg. 2006;117(1):10e-18e. doi:10.1097/01.prs.0000195081.39771.57

7. Cruse CW, Blevins PK, Luce EA. Naso-ethmoidorbital fractures. J Trauma. 1980;20(7):551-556. doi:10,1097/00005373-198007000-00003

8. Nguyễn Hùng Thẳng, Vũ Ngọc Lâm, Lê Đức Tuấn (2014), "Nghiến cứu đặc điểm lâm sàng gãy phức hợp mũi-sàng-ổ mắt một bên", Tạp chí Y Dược lâm sàng 108, (6), tr. 102-106.

9. Cultrara A, Turk JB, Har-EI G. Midfacial Degloving Approach for Repair of Naso-OrbitalEthmoid and Midfacial Fractures. Arch Facial Plast Surg. Published online March 1, 2004. doi:10.1001/archfaci.6.2.133

\title{
GIÁ TRI CỦA PHÂN LOAI IOTA ADNEX TRONG ĐÁNH GIÁ KHỐI U BUỒNG TRỨNG BẰNG SIÊU ÂM TẠI BỆNH VIỆN K
}

\author{
Nguyễn Duy Thái ${ }^{1}$, Dương Đức Hữu' ${ }^{1}$, \\ Hoàng Thị Vi Hương ${ }^{1}$, Ngô Đức Anh ${ }^{1}$, Nguyễn Văn Thi ${ }^{1}$

\section{SUMMARY \\ VALUE OF THE IOTA ADNEX CLASSIFICATION IN THE ULTRASOUND ASSESSMENT OF OVARIAN TUMORS AT K HOSPITAL} \\ Objectives: This study aim to evaluate the value
} TÓM TẮT

Mục tiêu: Nghiên cứu nhằm đánh giá giá trị của mô hình IOTA ADNEX trong siêu âm chẩn đoán mức độ lành tính - ác tính của khối u buồng trứng tại bệnh viện $K$. Đối tượng và phương pháp: Nghiền cứu được thực hiện trên 54 bệnh nhân trong khoảng thời gian từ tháng 12 năm 2020 đến tháng 05 năm 2021 tại bệnh viện $K$ với lâm sàng nghi ngờ u buồng trứng, được siêu âm trước phẫu thuật và thu thập số liệu theo mô hình IOTA ADNEX, được phẫu thuất với chẩn đoán sau phẫu thuât là u buồng trứng. Đối chiếu kết quả phẫu thuật, kết quả giải phẫu bệnh với mô hình IOTA ADNEX thu thập trước phẫu thuật. Từ đó đánh giá giá trị của mô hình IOTA ADNEX trong siêu âm chẩn đoán mức độ lành tính - ác tính u buồng trứng. Kết quả: Mô hình IOTA ADEX có CA 125 và mô hình IOTA ADNEX không có CA 125 có giá trịtốt trong chẩn đoán phân biệt u buồng trứng lành tính và ác tính với diện tích dưới đường cong ROC (Area under the curve - AUC) lần lướt là 0,977 và 0,968 . Ngưỡng cắt tối ưu của mô hình IOTA ADNEX có CA 125 và mô hình IOTA ADNEX không có CA125 lần lượt là 24,5 và 25,2 . Tại ngưỡng cắt tối ưu, cả hai mồ hình này đều có độ nhạy, độ đăc hiệu, giá trị dự báo dương tính, giá trị dự báo âm tính, độ chính xác lần lượt là 92,3\%, $96,8 \%, 96 \%, 93,8 \%, 94,7 \%$. Kết luận:Mô hình IOTA ADNEX có CA 125 và mô hình IOTA ADNEX không có CA 125 đều có giá trị cao và tương đồng trong chẩn đoán phân biệt u buồng trứng lành tính và ác tính ở bệnh viện K. Tư khóa: IOTA ADNEX, CA 125, u buồng trứng, siêu âm.

\section{${ }^{1}$ Bệnh viện $K$}

Chịu trách nhiệm chính: Nguyễn Duy Thái

Email: duythaibvk@gmail.com

Ngày nhận bài: 8.7.2021

Ngày phản biện khoa học: 31.8.2021

Ngày duyệt bài: 10.9.2021 of the IOTA ADNEX model in the diagnosis of benign malignant levels of ovarian tumors at $K$ hospital. Marterial and Methods: The propestive study was conducted on 54 patients from December 2020 to May 2021 at K hospital with clinical suspicion of ovarian tumor, were taken preoperative ultrasound and were collected data according to the IOTA ADNEX model, then were operated and diagnosed with ovarian tumor. The surgical and pathological results were compared with the IOTA ADNEX model data collected before surgery. Then the values of the IOTA ADNEX models in diagnosis ovarian tumors were evaluated. Results: Both the IOTA ADNEX model with CA 125 and the IOTA ADNEX model without CA 125 were very good for distinguishing between benign and malignant tumors with an Area under the curve (AUC) were 0,977 and 0,968 , respectively. The optimal cut - off point of the IOTA ADNEX model with CA 125 and the IOTA ADNEX model without CA 125 were 24,5 and 25,2 , respectively. At the optimal cut - off point, both predictive value, negative predictive value, accuracy of $92,3 \%, 96,8 \%, 96 \%, 93,8 \%, 94,7 \%$, respectively. Conclusion: Both the IOTA ADNEX model with CA 125 and the IOTA ADNEX model without CA 125 have high value and are similar in distinguishing between benign and malignant ovarian tumors at $K$ hospital.

Key words: IOTA ADNEX, CA 125, ovarian tumor, ultrasound.

\section{I. ĐặT VẤN ĐỀ}

U buồng trứng là một phát hiện phổ biến trong thực hành lâm sàng hàng ngày. ${ }^{1}$ Trong đó, two models had sensitivity, specificity, positive 
chẩn đoán tính lành ác của một khối u buồng trứng là một vấn đề thách thức bởi liên quan đến việc tiên lượng cũng như xác định kế hoạch điều trị thích hợp. ${ }^{2}$ Nếu u buồng trứng lành tính, phẫu thuật nội soi thường được áp dụng, nhưng nếu nghi ngờ ác tính, cần phải có một hướng can thiệp đầy đủ, đúng mức để cải thiện tỷ lệ sống và chất lượng cuộc sống. ${ }^{3}$

Siêu âm là phương pháp chẩn đoán hình ảnh đầu tay trong đánh giá u buồng trứng vì ít tốn kém, phổ biến và độ nhạy cao. ${ }^{2}$ Năm 2014, mô hình phân loại ADNEX do nhóm nghiên cứu khối u buồng trứng quốc tế - The International Ovarian Tumor Analysis (IOTA) xây dựng nhằm đánh giá nguy cơ ung thư buồng trứng được nhắc đến với độ chính xác cao và có nhiều ưu điểm hơn so với các mô hình trước đó. ${ }^{2}$

Mô hình IOTA ADNEX hiện nay đã được triển khai và áp dụng rộng rãi tại bệnh viện $K$. Tuy nhiên, chưa có một nghiên cứu nào tập trung về giá trị của mô hình này khi ứng dụng tại đây. Chính vì vậy, chúng tôi tiến hành nghiên cứu này nhằm đánh giá giá trị của phân loại IOTA ADNEX trong chẩn đoán phân biệt khối u buồng trứng lành tính và ác tính tại bệnh viện $\mathrm{K}$.

\section{II. ĐỐI TƯợNG VÀ PHƯƠNG PHÁP NGHIÊN CứU}

Đối tượng nghiên cứu. Các bệnh nhân có lâm sàng nghi ngờ u buồng trứng, được siêu âm trước phẫu thuật tai khoa Chẩn đoán hình ảnh bệnh viện $K$ với đầy đủ mô tả theo phân loại IOTA ADNEX, được xét nghiệm CA125 trước phẫu thuật, được phẫu thuật tại bệnh viện $\mathrm{K}$ và có kết quả sau phẫu thuật là u buồng trứng với kết quả giải phẫu bệnh đầy đủ, đồng ý tham gia vào nghiên cứu, có hồ sơ bệnh án đầy đủ.

Thời gian nghiên cứu: Từ tháng 12 năm 2020 đến tháng 05 năm 2021

Thiết kế nghiên cứu: Nghiên cứu mô tả cắt ngang, tiến cứu

Cỡ mẫu nghiên cứu: Mẫu thuận tiện

Phương pháp siêu âm. Tất cả các bệnh nhân tham gia vào nghiên cứu được tiến hành siêu âm phần phụ qua đường đường âm đạo. Siêu âm đường bụng được tiến hành khi nghi ngờ tổn thương ác tính, tổn thương kích thước lớn hoăc bênh nhân chưa quan hề tình dục.

Quy trình, phương pháp thu thập số liệu. Thu thập các thông tin về tuổi, tiền sử điều trị tại các trung tâm ung bướu (có/không), nồng độ CA125 trước phẫu thuât $(\mathrm{U} / \mathrm{ml})$, kết quả giải phẫu bệnh sau phẫu thuật. Các chỉ số siêu âm gồm: (1) dịch ổ bụng quan sát được ở ngoài túi cùng Douglas (có/không), bóng cản âm (có/không), đường kính khối u (mm), đường kính phần đặc lớn nhất $(\mathrm{mm})$, số nhú $(0,1,2,3$, $>3$ ), số thùy nhiều hơn 10 (có/không). Từ đó đánh giá được mức độ lành, ác tính theo mô hình IOTA ADNEX.

Các số liệu được thu thập và được xử lý theo phần mềm SPSS 20.0 và MedCalc 20.011.

\section{KẾT QUẢ NGHIÊN CỨU}

Có 54 bênh nhân tham gia vào nghiên cứu với 57 khối u bao gồm 31 khối u lành tính $(54,4 \%)$ và 26 khối u ác tính $(45,6 \%)$. Có 3 bệnh nhân có u buồng trứng hai bên chiếm tỷ lệ $5,6 \%$. Tuổi trung bình của quần thể nghiên cứu là $46,3 \pm 12,8$, nhỏ tuổi nhất là 21 và lớn tuổi nhất là 78 tuổi.

Bảng 1: Các chỉ số nghiên cứu theo mô hình phân loại IOTA ADNEX

\begin{tabular}{|c|c|c|c|c|c|}
\hline & $\begin{array}{c}\text { Lành tính } \\
(\mathrm{n}=\mathbf{3 1})\end{array}$ & $\begin{array}{l}\text { U giáp biên } \\
(n=5)\end{array}$ & $\begin{array}{c}\text { Giai đoạn I } \\
(n=8)\end{array}$ & $\begin{array}{c}\text { Giai đoạn } \\
\text { II-IV }(n=11)\end{array}$ & $\begin{array}{c}\text { Di căn } \\
(n=2)\end{array}$ \\
\hline \multicolumn{6}{|c|}{ Tiền sử điều trị tại trung tâm ung bướu (số lượng - \%) } \\
\hline Chưa điều trị & $31(100)$ & $5(100)$ & $8(100)$ & $10(90,9)$ & $1(50)$ \\
\hline Đã điêu tri & $0(0)$ & $0(0)$ & $0(0)$ & $1(9,1)$ & $1(50)$ \\
\hline \multicolumn{6}{|c|}{ Số thùy (số lượng - \%) } \\
\hline$<10$ thùy & $27(87,1)$ & $1(20)$ & $7(87,5)$ & $9(81,8)$ & $2(100)$ \\
\hline$\geq 10$ thùy & $4(12,9)$ & $4(80)$ & $1(12,5)$ & $2(18,2)$ & $0(0)$ \\
\hline \multicolumn{6}{|c|}{ Số nhú́ (số lượng - \%) } \\
\hline 0 & $21(67,6)$ & $1(20)$ & $1(12,5)$ & $0(0)$ & $0(0)$ \\
\hline 1 & $4(12.9)$ & $0(0)$ & $3(37,5)$ & $7(63,6)$ & $2(100)$ \\
\hline 2 & $2(6,5)$ & $0(0)$ & $2(25)$ & $2(18,2)$ & $0(0)$ \\
\hline 3 & $2(6,5)$ & $1(20)$ & $0(0)$ & $2(18,2)$ & $0(0)$ \\
\hline$>3$ & $2(6,5)$ & $3(60)$ & $2(25)$ & $0(0)$ & $0(0)$ \\
\hline \multicolumn{6}{|c|}{ Bóng cản (số lượng - \%) } \\
\hline Không & $24(77,4)$ & $3(60)$ & $8(100)$ & $11(100)$ & $2(100)$ \\
\hline Có & $7(22,6)$ & $2(40)$ & $0(0)$ & $0(0)$ & $0(0)$ \\
\hline \multicolumn{6}{|c|}{ Dịch ố bụng (số lượng - \%) } \\
\hline
\end{tabular}




\begin{tabular}{|c|c|c|c|c|c|}
\hline Không & $28(90,3)$ & $4(80)$ & $7(87,5)$ & $4(36,4)$ & $1(50)$ \\
\hline Có & $3(9,7)$ & $1(20)$ & $1(12,5)$ & $7(63,6)$ & $1(50)$ \\
\hline Tuổi & 21,9 & 67,4 & 67,2 & 156,2 & 120,3 \\
& $(17,4-26,2)$ & $(48,5-107)$ & $(62,5-148,5)$ & $(87,4-172,5)$ & $(76,4-120,3)$ \\
\hline CA125 & $39(34-48)$ & $45(34-54)$ & $50(38-55)$ & $57(49-65)$ & $58(53-58)$ \\
\hline Đường kính u (mm) & $73(64-96)$ & $87(73-93)$ & $83(71-92)$ & $67(54-85)$ & $40(38-40)$ \\
\hline Đường kính phần & 10 & 34 & 46 & 41 & 40 \\
đặc lớn nhất (mm) & $(6-11)$ & $(12-41)$ & $(41-66)$ & $(30-43)$ & $(38-40)$ \\
\hline
\end{tabular}

Bảng 2: Giá trị của mô hình IOTA ADNEXtại một số ngưỡng cắt

\begin{tabular}{|c|c|c|c|c|c|c|c|c|c|c|}
\hline \multirow{2}{*}{$\begin{array}{c}\text { Ngưỡng } \\
\text { cắt }\end{array}$} & \multicolumn{4}{|c|}{ IOTA ADNEX có CA 125 } & \multicolumn{4}{c|}{ IOTA ADNEX không Có CA 125 } \\
\cline { 2 - 11 } & Se & Sp & PPV & NPV & Acc & Se & Sp & PPV & NPV & ACC \\
\hline 5 & 96,1 & 71 & 73,5 & 95,6 & 82,5 & 96,2 & 67,7 & 71,4 & 95,5 & 80,7 \\
\hline 10 & 96,2 & 77,4 & 78,1 & 96 & 85,9 & 92,3 & 77,4 & 77,4 & 92,3 & 84,2 \\
\hline 15 & 92,3 & 87,1 & 85,7 & 93,1 & 89,5 & 92,3 & 87,1 & 85,7 & 93,1 & 89,5 \\
\hline $24,5\left(^{*}\right)$ & 92,3 & 96,8 & 96 & 93,8 & 94,7 & & & & & \\
\hline $25,2(* *)$ & & & & & & 92,3 & 96,8 & 96 & 93,8 & 94,7 \\
\hline
\end{tabular}

Se: độ nhạy; Sp: đô đặc hiệu; PPV: giá trị dự báo dương tính; NPV: giá trị dự báo âm tính; Acc: độ chính xác; $\left(^{*}\right)$ là điểm cắt tổi ưu của mô hình có sử dụng CA 125; (**) là điểm cắt tối ưu mô hình không sử dụng CA 125.

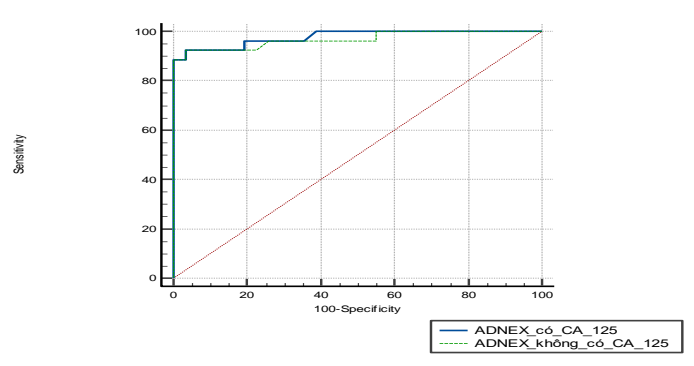

Biểu đồ 1: So sánh diện tích dưới đường cong ROC (AUC) của mô hình IOTA ADNEX có CA 125 và không có $C A 125$ trong chẩn đoán ung thư buồng trứng AUC của mô hình chẩn đoán IOTA ADNEX có sử dụng yếu tố CA 125 là 0,977 $(0,897-0,999)$. AUC của mô hình chẩn đoán IOTA ADNEX không có sử dụng yếu tố CA 125 là $0,968(0,884$ - 0,997). Kết quả kiểm định Hanlay - McNeil cho thây không có sự khác biệt AUC giữa hai mô hình này với $Z=1,007$ và $p=0,31$.

\section{BÀN LUÂNN}

Các dữ liệu trong nghiên cứu của chúng tôi cho thấy mô hình phân loại IOTA ADNEX tương đối tốt trong việc mô tả đặc điểm các khối u buồng trứng, nhưng cung cấp độ nhạy, độ đặc hiệu và độ chính xác cao trong việc ước tính độ ác tính của u buồng trứng.

Để chẩn đoán tính ác - lành u buồng trứng, giá trị AUC trong nghiên cứu của chúng tôi đạt 0,977 $(0,897$ - 0,999) đối với mô hình phân loại ADNEX có sử dụng CA 125 và $0,968(0,884$ $0,997)$ đối với mồ hình phân loại ADNEX không sử dụng CA 125 . Kết quả này tương đồng với nghiên cứu của Sayasneh và cộng sự năm 2016 (AUC $=0,937$ với mô hình ADNEX có CA 125 và
AUC $=0,925$ khi không có yếu tố CA 125) ${ }^{4}$.

Khi so sánh giá trị của mô hình IOTA ADNEX có $C A 125$ và không có $C A 125$ nhận thây không có sự khác biệt có ý nghĩa thống kê. ở điểm cắt $5 \%$ và $10 \%$ thì độ đăc hiêu, giá trị dự báo dương tính, giá trị dự báo âm tính và độ chính xác của mô hình có CA 125 cao nhe so với mô hình không có CA 125 . Nhưng ở điểm cắt $15 \%$ và điểm cắt tối ưu, nghiên cứu của chúng tôi cho thấy không có sự khác biệt giữa hai nhóm trên. Tất nhiên ở điểm cắt $5 \%$ và $10 \%$ có sự khác nhau nhe như vậy chứng tỏ rằng mô hình IOTA ADNEX không có CA 125 đã có chẩn đoán sai sót nhiều hơn so với mô hình IOTA ADNEX có CA 125 tai các điểm cắt này. Và rõ ràng, nếu chúng ta chẩn đoán sai càng ít, đăc biêtt là càng bỏ sót ít các tổn thương ác tính thì về mặt lâm sàng càng có lợi cho bệnh nhân, vì vậy mổ hình có $C A$ 125 có lợi hơn. Thực tế lâm sàng tại bệnh viện $K$, tất các các bệnh nhân đều được làm xét nghiệm CA 125 trước phẫu thuật. Tuy vậy, nhóm IOTA cũng khuyến cáo, nếu không có sắn xét nghiệm CA 125, có thể thực hiện hai bước: (1) sử dụng mô hình ADNEX không có CA 125 để đưa các bệnh nhân có nguy cơ ở ngưỡng mong đợi, thường là $10 \%$, (2) làm thêm xét nghiệm CA 125 nhằm phân định rõ nguy cơ ung thư theo các nhóm giai đoạn để có kế hoạch can thiệp cụ thể thích hợp tùy theo năng lực của cơ sở y tế. ${ }^{3}$ Bằng cách này, xét nghiệm nồng độ $\mathrm{CA} 125$ có thể được dùng một cách chọn lọc hơn, chỉ giới hạn trong những trường hợp có nguy cơ ung thư buồng trứng.

Nghiên cứu của Sayasneh và cộng sự năm 2016 cho thấy việc thiếu giá trị của CA 125 ít có 
tác động về việc phân biệt giữa các khối u lành tính và ác tính. ${ }^{4}$ Mức chênh lệch giữa giá trị AUC rất thấp giữa hai mô hình $A D N E X$ có $C A 125$ và ADNEX không có CA 125. Trong nghiên cứu của chúng tôi, sự khác biệt này không có ý nghĩa với $Z=1,007$ và $p=0,31$, tương tự như nghiên cứu của tác giả Sayasneh.

Các nghiên cứu về giá trị của mô hình IOTA ADNEX luôn cố gắng để tìm ra được ngưỡng cắt tôi ưu của nguy cơ ác tính. ${ }^{5}$ Việc tìm ra ngưỡng cắt tối ưu hợp lý giúp cho các bác sĩ xác định các khối u lành tính với đô đặc hiệu cao hơn, giúp tránh được các cuộc phẫu thuật không cân thiết các khối u lành tính, vì mục tiêu của điều trị bao gồm cả tăng chất lượng cuộc sống bệnh nhân bao gồm cả mong muốn sinh con. Tuy nhiên, nhóm IOTA cũng không đề xuất một cách chung thuật toán áp dụng với các ngưỡng cắt cố định, vì nhóm cho rằng, mô hình sẽ được sử dụng trong hoàn cảnh nào là rất quan trọng. ${ }^{4} \mathrm{Nghiên}$ cứu của chúng tôi cho thấy ngưỡng cắt tối ưu đối với mô hình IOTA ADNEX có CA 125 là $24,5 \%$ và với mô hình IOTA ADNEX không có Ca 125 là 25,2\%. Đồng thời, tại các ngưỡng cắt tối ưu, cả hai mô hình đều cho giá trị chẩn đoán nguy cơ ác tính giống nhau ( $\mathrm{Se}=92,3 \%, \mathrm{Sp}=$ $96,8 \%, \mathrm{PPV}=96 \%, \mathrm{NPV}=93,8 \%$ và $\mathrm{Acc}=$ $94,7 \%)$. Ngưỡng này cao hơn so với ngưỡng cắt thường sử dụng $(10 \%){ }^{3}$ thấp hơn nghiên cứu của Soo Young Jeong (47,3\%). ${ }^{5}$ Tuy nhiên, nghiên cứu của chúng tôi cho thây, ở ngương cắt $15 \%$, cả hai mô hình IOTA ADNEX đều có độ chính xác không có khác biệt nhiều với nghiên cứu của Van Castle. ${ }^{6}$ Do đó, chúng tôi cho rằng, giá trị ngưỡng cắt $15 \%$ có thể được đưa ra để xem xét đánh giá nguy cơ lành tính - ác tính của u buồng trứng tại bệnh viện $K$, giúp đưa ra chiến lược điều trị thích hợp.

\section{KẾT LUÂ̂N}

Mô hình phân loại IOTA ADNEX có CA 125 và IOTA ADNEX không có CA 125 đều có giá trị cao và tương đồng trong chẩn đoán phân biệt u buồng trứng lành tính - ác tính ở bệnh viện $\mathrm{K}$. Việc áp dụng mô hình IOTA ADNEX giúp cải thiện chẩn đoán trước phẫu thuật và góp phần đưa ra chiến lược điều trị thích hợp.

\section{TÀI LIÊU THAM KHẢO}

1. Foti PV, Attinà G, Spadola $S$, et al. MR imaging of ovarian masses: classification and differential diagnosis. Insights Imaging. 2016;7(1):21-41. doi:10.1007/s13244-015-0455-4

2. Nam G, Lee SR, Jeong $K$, Kim SH, Moon H-S, Chae HD. Assessment of different NEoplasias in the adneXa model for differentiation of benign and malignant adnexal masses in Korean women. Obstet Gynecol Sci. 2021; 64(3):293-299. doi: $10.5468 /$ ogs.21012

3. Lê Ngoc Diệp, Tô Mai Xuân Hông. Giá trị dự đoán độ ác tính u buồng trứng của mố hình IOTA ADNEX tai bênh viên Từ Dũ. Y học TP Hồ Chí Minh. 2019;23 (2):207-213.

4. Sayasneh A, Ferrara $L$, De Cock B, et al. Evaluating the risk of ovarian cancer before surgery using the ADNEX model: a multicentre external validation study. $\mathrm{Br} \mathrm{J}$ Cancer. 2016;115(5):542-548. doi:10.1038/bjc.2016.227

5. Jeong SY, Park BK, Lee YY, Kim T-J. Validation of IOTA-ADNEX Model in Discriminating Characteristics of Adnexal Masses: A Comparison with Subjective Assessment. JCM. 2020;9(6):2010. doi: $10.3390 / j \mathrm{~cm} 9062010$

6. Van Calster B, Van Hoorde $K$, Valentin $L$, et al. Evaluating the risk of ovarian cancer before surgery using the ADNEX model to differentiate between benign, borderline, early and advanced stage invasive, and secondary metastatic tumours: prospective multicentre diagnostic study. BMJ. 2014; 349(oct07 3):g5920-g5920. doi:10.1136/bmj.g5920

\section{QUẢN LÝ ĐIỀU TRI NGƯỜI BỆNH TĂNG HUYẾT ÁP TẠI PHƯờ'NG HỒNG HÀ, THÀNH PHỐ HẠ LONG, QUẢNG NINH NẰM 2021}

\section{Nguyễn Thị Linh*, Đặng Thị Thương**, Lê Vĩnh Giang***}

\section{TÓM TẮT}

*TTYT Thành phố Ha Long

**Viện Vệ sinh dịch tễ Trung ương

**Trường Đại học Y Hà Nội

Chịu trách nhiệm chính: Nguyễn Thị Linh

Email: nguyenlinh.khth87@gmail.com

Ngày nhận bài: 9.7.2021

Ngày phản biện khoa học: 6.9.2021

Ngày duyệt bài: 13.9.2021
Muc tiêu. Mô tả thức trang quản lý điều tri người bệnh tăng huyết áp từ 25 tuổi trở lên và một số yếu tố liển quan tại phướng Hồng Hà, thành phố Hạ Long, Quảng Ninh, năm 2021. Đối tượng và phương pháp nghiênn cứu. Nghiên cứu mô tả cắt ngang thực hiện trên 250 người trưởng thành đã được chẩn đoán mắc tăng huyết áp, đang sinh sống tai phường Hồng Hà, thành phố Hạ Long, tỉnh Quảng Ninh từ tháng 1/2021 đến tháng 09/2021. Kết quả. Trong tổng số bênh nhân THA được quản lý điều trị, $88,4 \%$ BN được quản lý hiệu quả. Mô hình hồi quy đa biến cho thấy có mối liên quan giữa tình trạng quản lý điều trị THA hiệu quả 